\title{
Computational Experience with Piecewise-Linear Relaxations for Petroleum Refinery Planning
}

\author{
Zaid Ashraf Rana, ${ }^{1}$ Cheng Seong Khor, ${ }^{1,2 *}$ \\ ${ }^{1}$ Chemical Engineering Department, Universiti Teknologi PETRONAS, 32610, Seri Iskandar, \\ Perak Darul Ridzuan, Malaysia. \\ ${ }^{2}$ Centre for Systems Engineering, Universiti Teknologi PETRONAS, 32610 Seri Iskandar, \\ Perak Darul Ridzuan, Malaysia.
}

*Corresponding author. E-mail: chengseong.khor@utp.edu.my; khorchengseong@gmail.com

\section{ABSTRACT}

Refinery planning optimization is a challenging problem as regards handling the nonconvex bilinearity mainly due to pooling operations in processes such as crude oil distillation and product blending. This work investigates the performance of several representative piecewiselinear (or piecewise-affine) relaxation schemes referred to as McCormick, bm, nf5, nf6t, and de (which is a new approach proposed based on eigenvector decomposition) that mainly give rise to mixed-integer optimization programs to convexify a bilinear term using predetermined univariate partitioning for instances of uniform and non-uniform partition sizes. Computational results show that applying these schemes give improved relaxation tightness than only applying convex and concave envelopes as estimators. Uniform partition sizes typically perform better in terms of relaxation solution quality and convergence behavior. It is also seen that there is a 
limit on the number of partitions that contributes to relaxation tightness, which does not necessarily correspond to a larger number of partitions, while a direct relation between relaxation size and tightness does not always hold for non-uniform partition sizes.

KEYWORDS: piecewise-linear relaxation; refinery planning; nonconvex; bilinear; nonlinear programming (NLP); mixed-integer linear programming (MILP)

\section{INTRODUCTION}

Optimization or mathematical programming models and tools are widely used in strategic and tactical planning of petroleum refinery operations. Major commercial refinery planning software include PIMS-AO (Aspen Technology), ${ }^{1}$ RPMS (Honeywell), ${ }^{2}$ GRTMPS (Haverly), ${ }^{3}$ and Spiral Plan (AVEVA). Existing use of such platforms largely involve linear programming (LP)-based techniques combined with heuristics and expert insights as well as rules-of-thumb to handle (including to simplify) the inherently nonlinear refinery processing behavior. On the other hand, it is deemed as to use rigorous planning models ${ }^{4}$ if they are not able to adequately represent the intended process details that possibly involve nonconvex nonlinearity towards obtaining globally optimal solutions,${ }^{5}$ whose features likely vary from one plant to another. ${ }^{6-7}$ In this regard, there is interest for refineries to develop their own planning models, ${ }^{8}$ but which necessitates customizing solution strategies rather than relying on off-the-shelf solvers particularly to handle the presence of nonconvexity.

There is ongoing effort to capture the complexity of refinery operations in formulating suitable planning optimization models. ${ }^{9-10}$ A chief interest is to improve the operational representation of a crude distillation units (CDU), which is a main refining process to separate crude oil mixtures into different fractions (e.g., naphtha, light and heavy distillates, bottom residue) 
based on boiling point differences. The complex nature of crude oil as feed and distillation operation with multiple inflows (e.g., reflux) and outflows (e.g., side streams) of material and energy make CDU modeling challenging. ${ }^{11-12}$

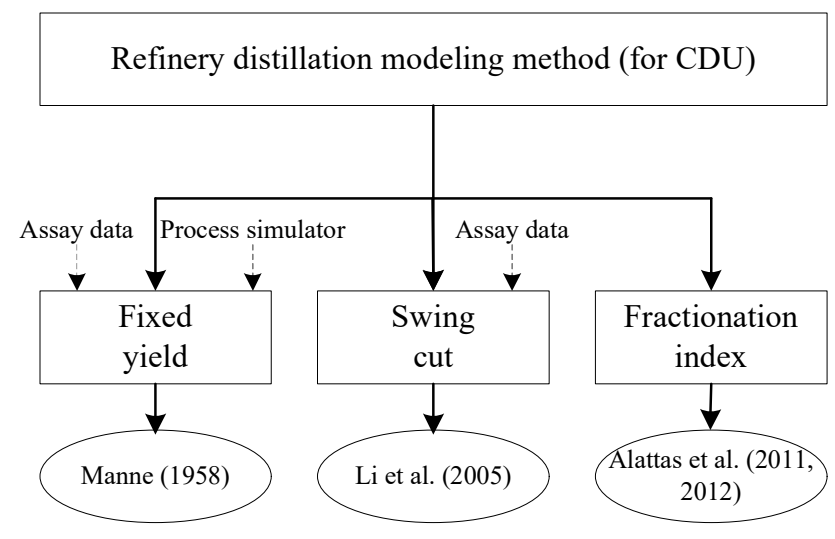

Figure 1. Modeling methods for petroleum refinery distillation cutpoints (mainly for CDU).

There are several modeling approaches to estimate CDU outlet fractions or distillates; also called crude oil cuts or simply "cuts". The methods include fixed yield, swing cut, and fractionation index as summarized in Figure 1 in terms of their input data and representative (seminal) work. Fixed yield is a basic method to represent CDU distillates as constant values specified based on historical data (e.g., crude assay reports), experience (i.e., expert advice), or process simulation models. Swing cut is more detailed by estimating a small portion of the yields (i.e., the "swing cut") that overlaps between adjacent cuts either represented as a parameter or determined as a decision variable. A potentially more accurate and physicallybased technique uses fractionation index ${ }^{13}$ that incorporates phase equilibrium and relative volatility to represent component distributions of the CDU cuts. Table 1 compares key attributes of these techniques including application examples. It is noteworthy that an emerging approach is to adopt suitable surrogate models to represent the yields (e.g., by improving the swing cuts prediction). ${ }^{14}$ 
Table 1. Methods to model refinery distillation yields of crude oils.

\begin{tabular}{|c|c|c|c|}
\hline Method & $\begin{array}{l}\text { Relation } \\
\text { Type }\end{array}$ & Feature & Application \\
\hline Fixed yield & Linear & $\begin{array}{l}\text { Simple; amenable to large scale models } \\
\text { (especially LP); can cater for different } \\
\text { operating modes }\end{array}$ & $\begin{array}{l}\text { Simulation }{ }^{15} \text {, planning optimization } \\
\left(\mathrm{LP},{ }^{15} \mathrm{NLP},{ }^{76} \mathrm{MINLP}^{17}\right) \text {, scheduling } \\
\text { optimization }\left(\mathrm{MILP}^{8}\right)\end{array}$ \\
\hline Swing cut & $\begin{array}{l}\text { Linear or } \\
\text { nonlinear }\end{array}$ & $\begin{array}{l}\text { More accurate than fixed yield; can } \\
\text { represent multiple operating modes }{ }^{18}\end{array}$ & $\begin{array}{l}\text { Planning optimization (LP }{ }^{19}, \text { MILP, } \\
\text { NLP, }{ }^{14,20-26} \text { MINLP }^{27}\end{array}$ \\
\hline $\begin{array}{l}\text { Fractionation } \\
\text { index }\end{array}$ & Nonlinear & $\begin{array}{l}\text { High accuracy (considers relative volatility } \\
\text { and phase equilibrium) }\end{array}$ & $\begin{array}{l}\text { Planning optimization (NLP, } \\
\text { MINLP) }\end{array}$ \\
\hline
\end{tabular}

This work considers a swing cut-based refinery planning nonlinear programming (NLP) model such as that based on Li et al. ${ }^{23}$ Existing work involves modeling swing cuts largely by using empirical correlations ${ }^{23}$ or determining a temperature range to define the associated swing cuts. ${ }^{14}$ Solution techniques include applying LP relaxations based on McCormick's convex and concave envelopes ${ }^{30-31}$ to improve bounds tightening within a gradient-based local NLP solver ${ }^{5}$ as well as using a branch and bound-based global solver such as ANTIGONE ${ }^{32}$ to perform integrated refinery and petrochemicals planning. ${ }^{33}$

Reduced computational times are reported for solving large-scale pooling problems using piecewise-linear relaxations within global optimization framework. ${ }^{34-35}$ Wicaksono and Karimi ${ }^{36}$ and Gounaris et al. ${ }^{37}$ propose several piecewise-linear relaxation schemes using $a b$ initio univariate and bivariate partitioning ${ }^{38}$ to handle nonconvex bilinear terms. A survey of developments on such relaxation methods can be found in Misener and Floudas. ${ }^{39}$ A related recent theoretical development is reported in Nagarajan et al. ${ }^{40}$

Several global optimization methods for handling bilinear functions are reported in the literature. ${ }^{36,41-46}$ A common technique uses spatial branch-and-bound framework ${ }^{47}$ which is similar to the class of branch-and-bound methods developed for integer optimization problems (e.g., pure integer linear program (ILP) or mixed-integer linear program (MILP) $)^{48}$ with a main 
difference in that spatial branch-and-bound performs branching on continuous rather than discrete variables. The main challenges in spatial branch-and-bound involve devising and applying effective branching strategies in addition to efficient procedures for obtaining tight lower and/or upper bounds. An implementation of this framework is available in the BARON solver, which incorporates a branch-and-reduce technique to perform variable range reduction based on Lagrangean multipliers. ${ }^{49-51}$

Other global optimization approach proposed includes a branch-and-contract algorithm for univariate concave, bilinear, and linear fractional functions that emphasizes reducing the number of branch-and-bound search tree nodes through a contraction operator. ${ }^{52}$ The relatively recent global optimization solver of ANTIGONE (commercial version of GloMIQO) offers capabilities based on advances in piecewise-linear (or -affine) relaxation algorithms. ${ }^{53}$ Computational comparisons are available for a number of global optimization codes on benchmark problems. ${ }^{37,54}$

Much effort focusses on constructing convex relaxations for factorable nonconvex NLP problems. This class of problems exclusively involves factorable functions, which can be expressed as recursive sums and products of univariate functions. ${ }^{55}$ Symbolic reformulation techniques have been proposed to transform an arbitrary factorable nonconvex program into an equivalent standard form in which all nonconvex terms are expressed a special nonlinear terms such as bilinear and concave univariate terms. ${ }^{56}$ These special nonlinear terms form the building blocks for factorable problems that abound in a wide range of disciplines including chemical engineering. In addition to those mentioned earlier, many problems in (chemical) process systems engineering such as design, operation, and control fall within this scope. ${ }^{57-60}$ 
Thus by addressing bilinear functions in this work, we are essentially addressing the much wider class of nonconvex factorable programs.

Our work contributes by conducting computational comparisons on several piecewise-linear relaxation schemes with potentially advantageous performance. Our study implements the schemes on a representative refinery planning nonconvex (bilinear) NLP model which involves distillation, conversion (reaction), and blending operations. We also compare the results with commercial global solvers (BARON and ANTIGONE). The rest of the paper consists of a brief description of the planning model followed by its reformulated relaxation versions that include a new proposed scheme based on separable programming.

\section{PROBLEM DESCRIPTION ON REFINERY PLANNING MODEL}

The computational study considers a petroleum refinery planning problem with its configuration shown in Figure 2 and described as follows (with the symbols denoted in the diagram stated in parentheses). A crude oil mixture enters CDU that separates this feed stream into five cuts, namely gross overhead (GO), heavy naphtha (HN), light distillate (LD), heavy distillate (HD), and bottom residue (BR). The lighter CDU cuts of GO and $\mathrm{HN}$ are blended with FCC gasoline (FGas) and MTBE (methyl tert-butyl ether, which functions as a gasoline additive) in gasoline blending unit (GB) to produce two gasoline grades represented by streams 90G and 93G (with octane numbers of at least 90 and 93, respectively). The heavier CDU cuts of LD and HD are blended in diesel blending unit (DB) to produce two diesel grades represented by streams $-10 \mathrm{D}$ and $0 \mathrm{D}$ (with pour points of at most $-10^{\circ} \mathrm{C}$ and $0^{\circ} \mathrm{C}$, respectively). The BR cut from CDU is fed to FCC that produces four outlets, namely a stream containing components with two to four carbon atoms (C24), FCC gasoline (FGas), total gas oil (TGO), 
and coke (COKE). C24 is sold as a valuable product while FGas is fed to GB. A portion of TGO is recycled to mix with BR with the remaining sold as FCC heavy oil (FHO). The coke produced is consumed internally in the refinery as a fuel source.

Mathematical formulation for the refinery planning NLP model is provided as Supporting Information.

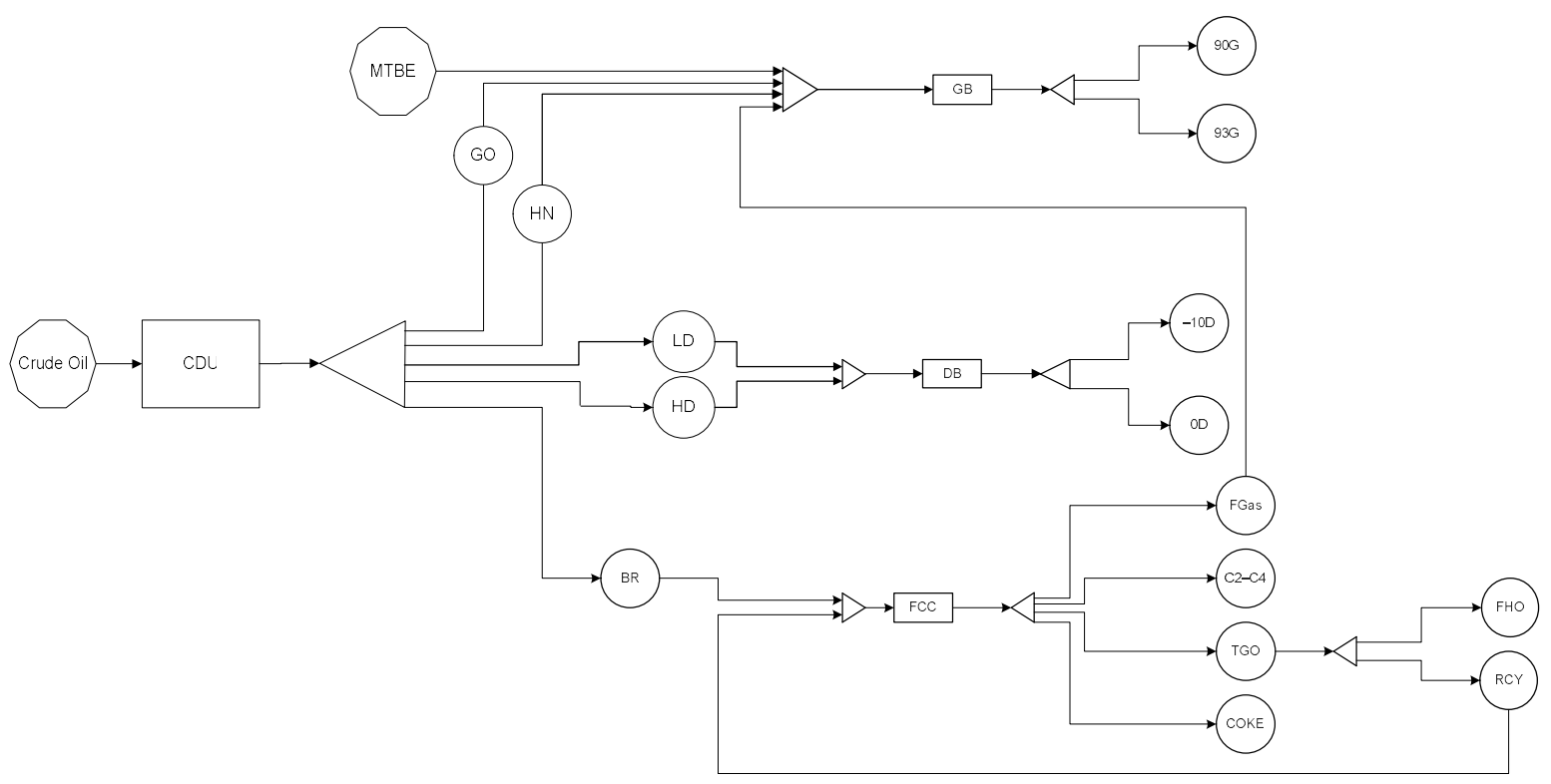

Figure 2. Flowsheet configuration for refinery planning model (NLP).

\section{COMPUTATIONAL EXPERIMENTS}

This section presents our computational experiment results to investigate the performance of several representative piecewise-linear relaxation methods applied to nonconvex bilinear terms in the foregoing refinery planning NLP model. Table 2 lists the bilinear terms and their number of occurrences in the model. Unless otherwise stated, we reformulate the model obtain its relaxations by implementing univariate partitioning. 
Table 2. Bilinear terms in refinery planning NLP model.

\begin{tabular}{cc}
\hline Bilinear Term & Count \\
\hline$L_{u} W_{p}$ & 5 \\
$L_{u} Y_{f}$ & 4 \\
$\operatorname{Conv} \times \operatorname{Conv}=(\operatorname{Conv})^{2}$ & 4 \\
$M W_{p} \times M W_{p}=M W_{p}^{2}$ & 4 \\
$P r_{j, p} F_{p, g}^{\text {iprod }}$ & 4 \\
\hline \multicolumn{1}{c}{ Total } & 21 \\
\hline
\end{tabular}

\subsection{Reformulation as Relaxation Models}

The bilinear terms in the refinery planning model are identified and replaced with new singleterm variables in reformulating their associated constraints to obtain a convexified model (either as LP or MILP). We apply the following relaxation methods for the reformulation (which are mostly referred to by the names used in their original publications): ${ }^{36-37}$ convex and concave envelopes relaxation called mc scheme,${ }^{55}$ a big- $M$ relaxation called bm scheme, ${ }^{36-37}$ two incremental cost relaxations called nf5 and nf6t schemes, ${ }^{36-37}$ and a decomposition in eigenvector directions scheme called de. The reformulated relaxed models for these schemes are given in Supporting Information as applied to the bilinear term $L_{u} W_{p}$ in equation B26). The resulting relaxed models of LP (for mc scheme) and MILP (for bm, nf5, and nf6t schemes) are solved using GAMS 30.3.0/CPLEX 12. Figure 3 shows a flowchart on the procedure used in our computational comparison study. 


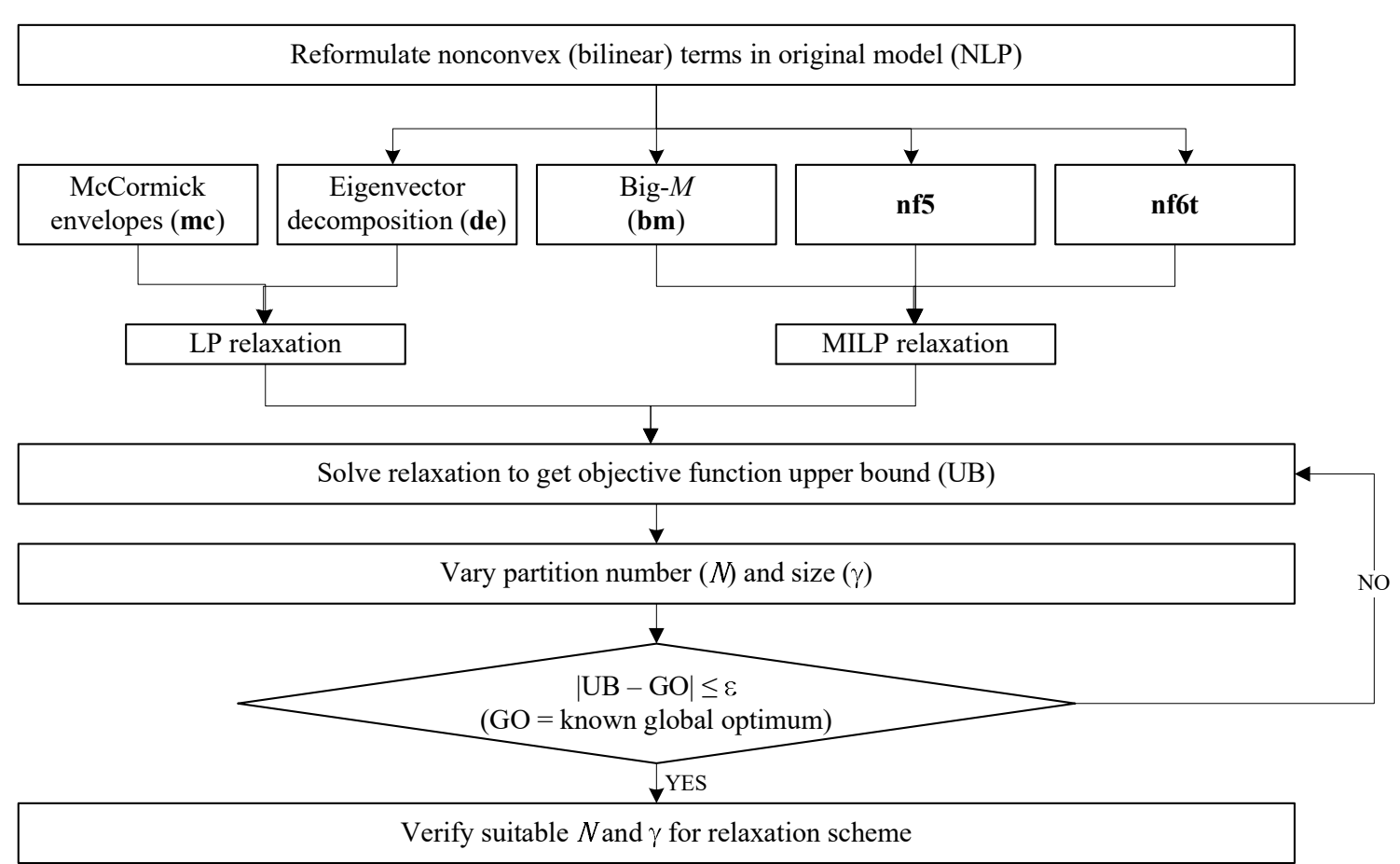

Figure 3. Procedure for investigating performance of piecewise-linear relaxation schemes on a nonconvex bilinear refinery planning NLP model.

The first scheme is the McCormick relaxation approach that involves representing (and replacing) a bilinear term by constructing its convex and concave envelopes as shown in the following constraints for the term $W_{p}$ in bilinear variable $L_{u} W_{p}$ :

$$
\begin{aligned}
& \omega_{u, p} \geq L_{u}^{\mathrm{LO}} W_{p}+L_{u} W_{p}^{\mathrm{LO}}-L_{u}^{\mathrm{LO}} W_{p}^{\mathrm{LO}}, u=\mathrm{CDU}, \forall p \\
& \omega_{u, p} \geq L_{u}^{\mathrm{UP}} W_{p}+L_{u} W_{p}^{\mathrm{UP}}-L_{u}^{\mathrm{UP}} W_{p}^{\mathrm{UP}}, u=\mathrm{CDU}, \forall p \\
& \omega_{u, p} \leq L_{u}^{\mathrm{UP}} W_{p}+L_{u} W_{p}^{\mathrm{LO}}-L_{u}^{\mathrm{UP}} W_{p}^{\mathrm{LO}}, u=\mathrm{CDU}, \forall p \\
& \omega_{u, p} \leq L_{u}^{\mathrm{LO}} W_{p}+L_{u} W_{p}^{\mathrm{UP}}-L_{u}^{\mathrm{LO}} W_{p}^{\mathrm{UP}}, u=\mathrm{CDU}, \forall p
\end{aligned}
$$

where $\omega_{u, p}$ is reformulated bilinear term $L_{u} W_{p}$ while superscripts LO and UP are lower and upper bounds, respectively of the bilinear term. 
The second scheme called $\mathbf{b m}$ is of the big- $M$ relaxation type that is reported to give tighter relaxation than $\mathbf{m c}$ scheme ${ }^{37}$ by dividing the domain of a partitioned bilinear variable term into $N$ segments (i.e., partitions) and applying overestimators and underestimators for each segment $n$ where $n=1,2, \ldots N$ as shown in the following for the bilinear variable term $W_{p}$ :

$$
\begin{gathered}
\sum_{n} \lambda_{n, p}=1, \quad \forall p \\
W_{p} \geq W_{p}^{\mathrm{LO}}+\left(k_{n-1, p}-W_{p}^{\mathrm{LO}}\right) \lambda_{n, p}, \quad \forall n, p \\
W_{p} \leq W_{p}^{\mathrm{UP}}-\left(W_{p}^{\mathrm{UP}}-\mathrm{k}_{n, p}\right) \lambda_{n, p}, \quad \forall n, p \\
\omega_{u, p} \geq W_{p} L_{u}^{\mathrm{LO}}+k_{n-1, p}\left(L_{u}-L_{u}^{\mathrm{LO}}\right)-M_{p}\left(1-\lambda_{n, p}\right), \quad u=\mathrm{CDU}, \forall n, p \\
\omega_{u, p} \geq W_{p} L_{u}^{\mathrm{UP}}+k_{n, p}\left(L_{u}-L_{u}^{\mathrm{UP}}\right)-M_{p}\left(1-\lambda_{n, p}\right), \quad u=\mathrm{CDU}, \forall n, p \\
\omega_{u, p} \leq W_{p} L_{u}^{\mathrm{UP}}+k_{n-1, p}\left(L_{u}-L_{u}^{\mathrm{UP}}\right)+M_{p}\left(1-\lambda_{n, p}\right), \quad u=\mathrm{CDU}, \forall n, p \\
\omega_{u, p} \leq W_{p} L_{u}^{\mathrm{LO}}+k_{n, p}\left(L_{u}-L_{u}^{\mathrm{LO}}\right)+M_{p}\left(1-\lambda_{n, p}\right), \quad u=\mathrm{CDU}, \forall n, p
\end{gathered}
$$

where $\lambda_{n, p}$ is binary variable that is equal to one if variable $W_{p}$ is activated in subdomain $n$ for CDU fraction $p, k_{n, p}$ is grid point of segment $n$ for fraction $p$, and $M_{p}$ is big- $M$ parameter for $p$. The resulting relaxed model gives rise to an MILP.

The third scheme, nf5 incrementally builds on the values of a partitioned bilinear variable term through successive segmenting (hence called an incremental cost relaxation type), resulting in a tighter relaxation than the bm scheme and similarly leads to an MILP relaxed model as follows for the bilinear variable term $W_{p}$ :

$$
W_{p}=W_{p}^{\mathrm{LO}}+\sum_{n} q_{n} d U_{n, p}, \quad u=\mathrm{CDU}, \forall p
$$




$$
\begin{gathered}
d U_{n, p} \geq \theta_{n, p}, \quad \forall n<N, p \\
d U_{n, p} \leq \theta_{n-1, p}, \quad \forall n>1 \\
\omega_{u, p}=W_{p}^{\mathrm{LO}} L_{u}+W_{p} L_{u}{ }^{\mathrm{LO}}-L_{u}{ }^{\mathrm{LO}} W_{p}^{\mathrm{LO}}+\sum_{n} q_{n} d Q_{n, p}, \quad u=\mathrm{CDU}, \forall p \\
d Q_{n, p} \geq\left(W_{p}^{\mathrm{UP}}-W_{p}^{\mathrm{LO}}\right) d U_{n, p}+W_{p}-W_{p}^{\mathrm{UP}}, \quad n=1, \forall p \\
d Q_{n, p} \geq d V_{n, p}, \quad \forall n<N, p \\
d Q_{n, p} \geq\left(W_{p}^{\mathrm{UP}}-W_{p}^{\mathrm{LO}}\right)\left(d U_{n, p}-\theta_{\mathrm{n}-1}\right)+d V_{n-1, p}, \quad \forall n>1, p \\
d Q_{n, p} \leq W_{p}-W_{p}^{\mathrm{LO}}, \quad n=1, \forall n, p \\
d Q_{n, p} \leq\left(W_{p}^{\mathrm{UP}}-W_{p}^{\mathrm{LO}}\right)\left(d U_{n, p}-\theta_{n}\right)+d V_{n, p}, \quad \forall n<N, \forall p \\
d Q_{n, p} \leq d V_{n-1, p}, \quad \forall n>1, \forall p \\
d Q_{n, p} \leq\left(W_{p}^{\mathrm{UP}}-W_{p}^{\mathrm{LO}}\right) d U_{n, p}, \quad n=N, \forall p
\end{gathered}
$$

where $\theta_{n, p}$ is binary variable that is equal to one if variable $W_{p}$ is activated in subdomain $n$ for CDU fraction $p, q_{n}$ is partition length for variable $L_{u}$ defined as $q_{n}=\left((n / N)^{\gamma}-\right.$ $\left.((n-1) / N)^{\gamma}\right)\left(W_{p}^{\mathrm{UP}}-W_{p}^{\mathrm{LO}}\right)$, and the following three sets of continuous variables comprising $d U_{n, p} \in[0,1], d Q_{n, p} \in\left[0, W_{p}^{\mathrm{UP}}-W_{p}^{\mathrm{LO}}\right], n=1,2, \ldots, N$, and $d V_{n} \in\left[0, W_{p}^{\mathrm{UP}}-\right.$ $\left.W_{p}^{\mathrm{LO}}\right], n=1,2, \ldots, N-1$.

The fourth scheme, nf6t also belongs to the incremental cost relaxation type as based on the foregoing nf5 scheme, but the latter entails a smaller size at the expense of reduced tightness. ${ }^{37}$ Implementing nf6t scheme to reformulate the same bilinear term $W_{p}$ comprises equations (12), (13), (14), (15), and (23) to (26):

$$
d W_{n, p} \geq\left(W_{p}^{\mathrm{UP}}-W_{p}^{\mathrm{LO}}\right) d U_{n}+W_{p}-W_{p}^{\mathrm{UP}}, \quad \forall n, p
$$




$$
\begin{gathered}
d W_{n, p} \leq W_{p}-W_{p}^{\mathrm{LO}}, \quad n=1, \forall p \\
d W_{n, p} \leq d W_{n-1, p}, \quad \forall n>1, p \\
d W_{n, p} \leq\left(W_{p}^{\mathrm{UP}}-W_{p}^{\mathrm{LO}}\right) d U_{n, p}, \quad \forall n, p
\end{gathered}
$$

The formulation for a proposed fifth scheme, de is presented in the following for the bilinear variable $L_{u} W_{p}$ :

$$
\begin{gathered}
L_{u} W_{p}=\omega^{\xi}-\omega^{\eta} \\
\xi=\left(L_{u}+W_{p}\right) / 2, \quad \forall u, p \\
\eta=\left(L_{u}-W_{p}\right) / 2, \quad \forall u, p
\end{gathered}
$$

where $\omega^{\xi}=\xi^{2}$ and $\omega^{\eta}=\eta^{2}$. Bounding overestimators and underestimators in $\xi$-direction are as follows:

$$
\begin{gathered}
\omega^{\xi} \geq 2 \overline{\xi_{J}} \xi-\overline{\xi_{J}^{2}}, \quad j=1, \cdots, M^{\xi} \\
\omega^{\xi} \leq \sum_{n} \xi_{n}^{2} \lambda_{n}^{\xi} \\
\xi=\sum_{n} \xi_{n} \lambda_{n}^{\xi} \\
\sum_{n} \lambda_{n}^{\xi}=1 \\
\text { SOS: } \lambda_{n}^{\xi} \quad n=0, \cdots, N^{\xi}
\end{gathered}
$$

Bounding overestimators and underestimators in $\eta$-direction are as follows:

$$
\begin{gathered}
\omega^{\eta} \geq 2 \overline{\eta_{J}} \eta-\overline{\eta_{J}^{2}}, \quad j=1, \cdots, M^{\eta} \\
\omega^{\eta} \leq \sum_{n} \eta_{n}^{2} \lambda_{n}^{\eta}
\end{gathered}
$$




$$
\begin{gathered}
\eta=\sum_{n} \eta_{n} \lambda_{n}^{\eta} \\
\sum_{n} \lambda_{n}^{\eta}=1 \\
\text { SOS: } \lambda_{n}^{\eta}, \quad n=0, \cdots, N^{\eta}
\end{gathered}
$$

\subsection{Computational Results}

The original refinery planning model is solved using BARON and ANTIGONE in which both report the same $\varepsilon$-global optimal value of US\$75,494 for the objective function (profit maximization). The reformulated relaxed models as MILP are solved using CPLEX (12.10.0) to obtain an upper bound of US\$98,547 for the maximization objective function.

Each of the relaxed models is solved by varying the associated number of partitions $n$ (for $n=$ $1,2,3,4,5,7,10,15,20,25)$ and partition size $\gamma($ for $\gamma=0.25,0.5,1,1.5,2,3,4)$ with the model performance shown in Figure 4 to Figure 10. We observe that as $\gamma$ is decreased to values less than unity (in which $\gamma=1$ corresponds to uniform partitioning), the magnitude of the difference between an upper bound (given by relaxed model solution) and a global optimum generally increases in the order of $\mathbf{n f 5}<\mathbf{n f 6 t}<\mathbf{d e}<\mathbf{b m}$ (see Figure 4 to Figure 6). On the other hand, as $\gamma$ is increased to values greater than unity, the magnitude difference between an upper bound and a global optimum generally increases in the order of $\mathbf{b m}<\mathbf{d e}<\mathbf{n f 5}<\mathbf{n f 6 t}$ (see Figure 7 to Figure 10). A convexified model using nf5 is tighter than nf6t but at the expense of a larger size while that of $\mathbf{b m}$ as well as de varies with respect to $\gamma$, which is consistent with reported results. ${ }^{37}$ In terms of the relaxed model sizes which are in the order of bm $<$ de $<$ nf6t $<$ nf5, a larger model size typically results in a tighter relaxation. ${ }^{37}$. Our study finds that as the partitioning levels $(n)$ and sizes $(\gamma)$ are increased, bm (which is smallest in size) shows tighter relaxation compared to the other schemes. 
We consider a convergence indicator for a resulting relaxation model that measures the distance traversed by a relaxed solution towards attaining an $\varepsilon$-global optimal solution. This indicator is evaluated at a certain partition level $n$ with respect to a corresponding upper bound (for $n=1$ ) and a lower bound (for $n=N$ ) as tabulated in Table 3. For $1 \leq N \leq 10$, an average value of about 90 percent is estimated as also depicted by the slope (steepness) of the convergenceindicating curves in Figure 4 to Figure 10 . In contrast, for $N \geq 10$, a significantly lower average value of approximately 10 percent is indicated. Therefore, a limit on the number of partitions can be observed that exhibits a certain relaxation tightness, beyond which no increased tightness or performance improvement is obtained (notwithstanding the increased model size and computational load).

Table 3: Convergence indicator of $\mathbf{b m}, \mathbf{d e}, \mathbf{n f 5}$, and $\mathbf{n f 6 t}$ relaxation schemes with partition size variation.

\begin{tabular}{crr}
\hline$\gamma$ & $\begin{array}{c}\text { Convergence indicator (\%) } \\
\text { for } 1 \leq N \leq 10\end{array}$ & $\begin{array}{c}\text { Convergence indicator (\%) } \\
\text { for } N \geq 10\end{array}$ \\
\hline 0.25 & 92 & \\
0.5 & 93 & 8 \\
1 & 90 & 7 \\
1.5 & 91 & 10 \\
2 & 88 & 9 \\
3 & 93 & 12 \\
4 & 80 & 7 \\
Average & 90 & 20 \\
\hline
\end{tabular}

To investigate the performance of uniform and non-uniform partition sizes, we determine an average relative difference or deviation of a relaxed solution from an $\varepsilon$-global optimal solution for each value of $\gamma$ and $n$. Figure 11 shows a lowest deviation (about 33 percent) for uniform partitioning $(\gamma=1)$ compared to other non-uniform partition sizes, thereby indicating better solution quality for the former in employing a piecewise-linear relaxation scheme as is consistent with the literature. ${ }^{37-38}$ 




Figure 4. Convergence-indicating curves of $\mathbf{b m}, \mathbf{d e}, \mathbf{n f 5}$, and $\mathbf{n f 6 t}$ relaxation schemes for $\gamma=$ 0.25 .

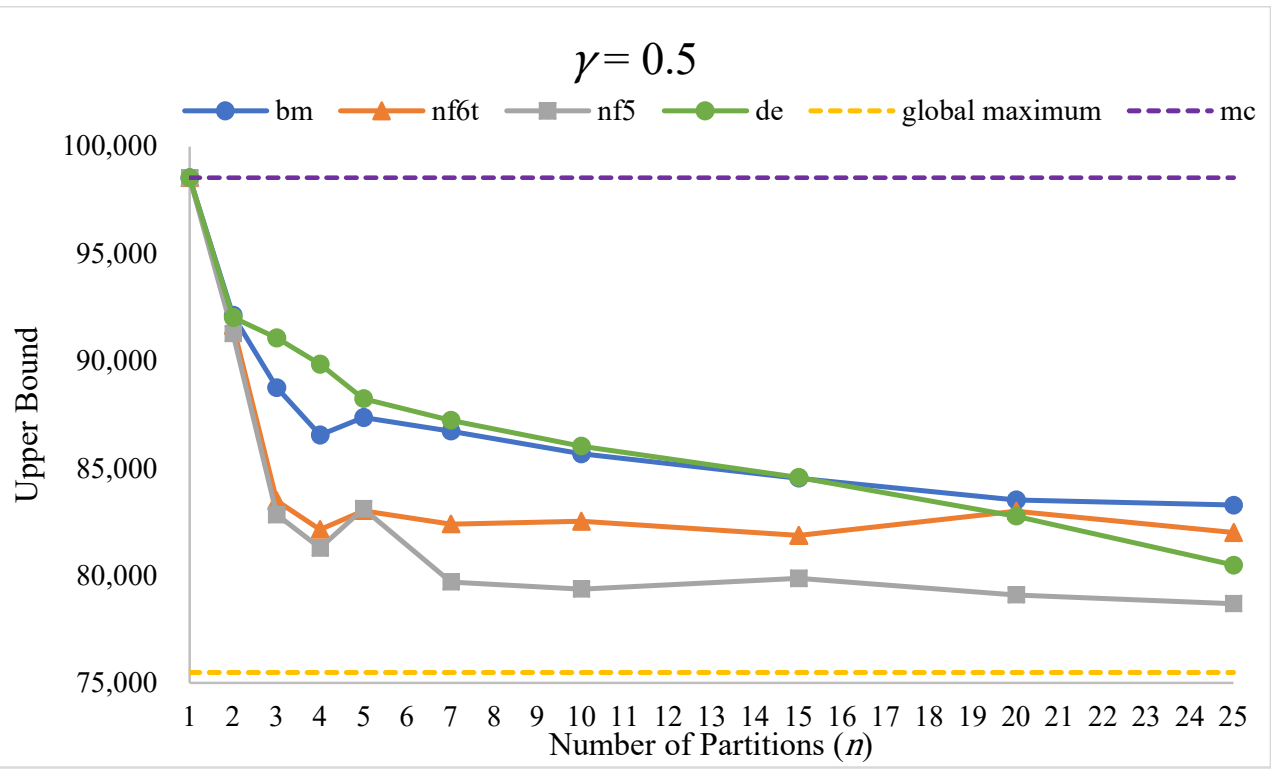

Figure 5. Convergence-indicating curves of $\mathbf{b m}$, de, $\mathbf{n f 5}$, and $\mathbf{n f 6 t}$ relaxation schemes for $\gamma=$ 0.5 . 




Figure 6. Convergence-indicating curves of $\mathbf{b m}, \mathbf{d e}, \mathbf{n f 5}$, and $\mathbf{n f 6 t}$ relaxation schemes for $\gamma=$

1.

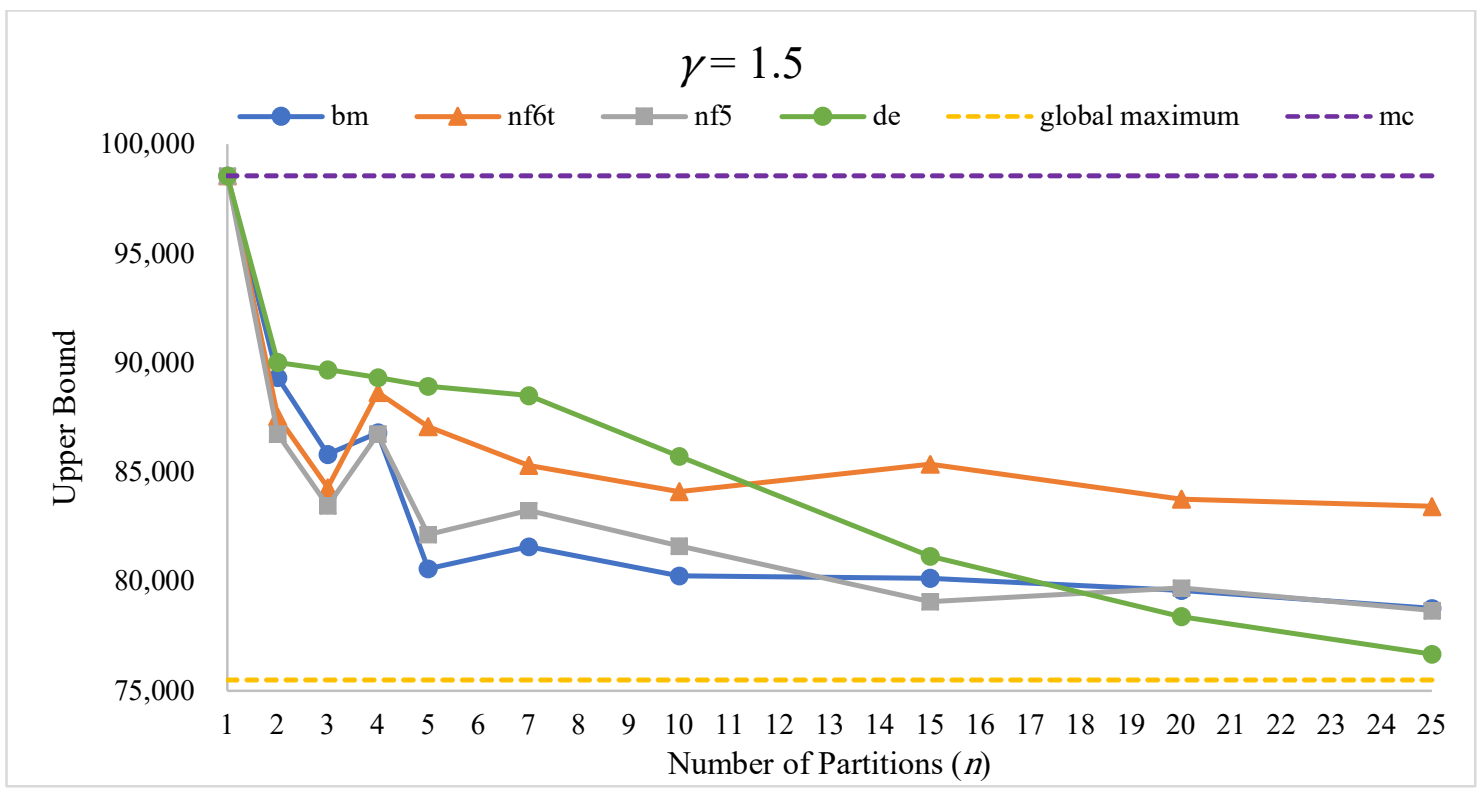

Figure 7. Convergence-indicating curves of $\mathbf{b m}, \mathbf{d e}, \mathbf{n f 5}$, and $\mathbf{n f 6 t}$ relaxation schemes for $\gamma=$ 1.5 . 


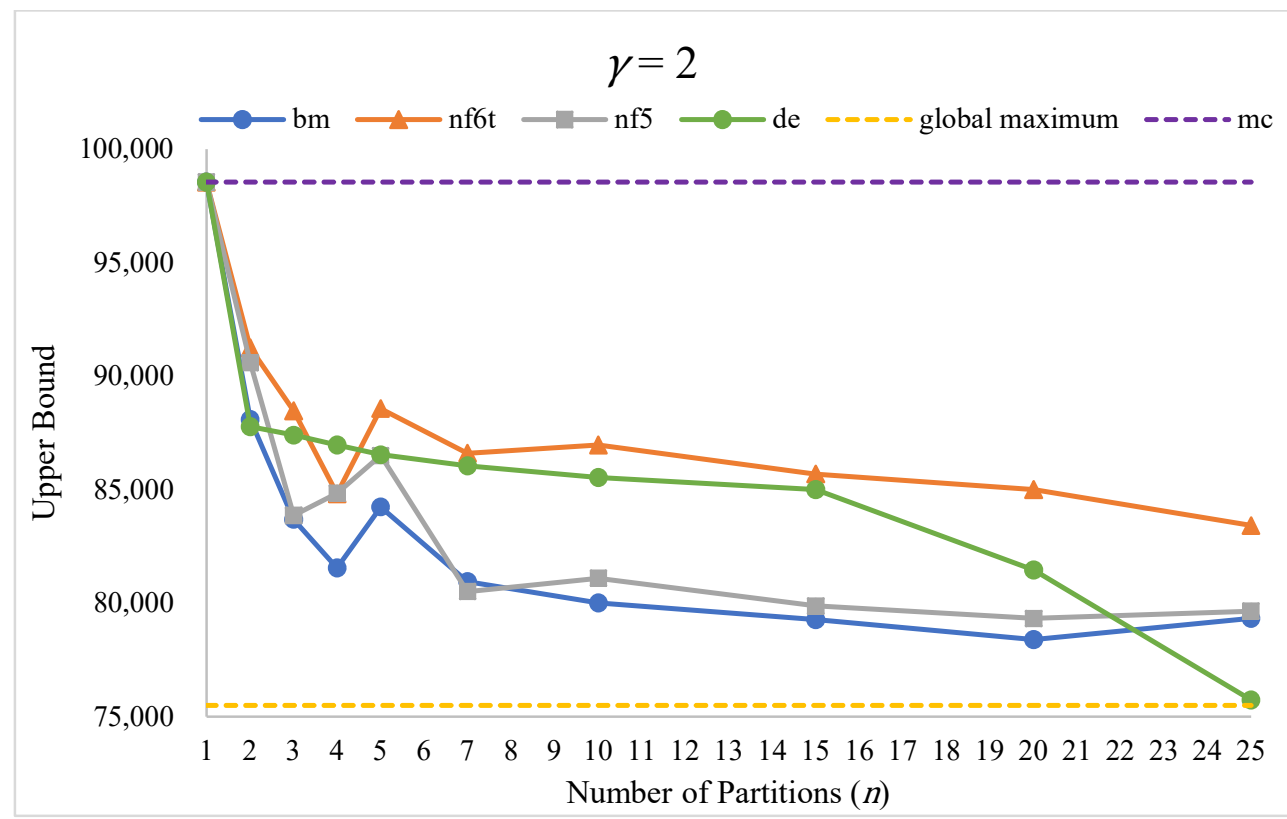

Figure 8. Convergence-indicating curves of $\mathbf{b m}, \mathbf{d e}, \mathbf{n f 5}$, and $\mathbf{n f 6 t}$ relaxation schemes for $\gamma=$ 2.

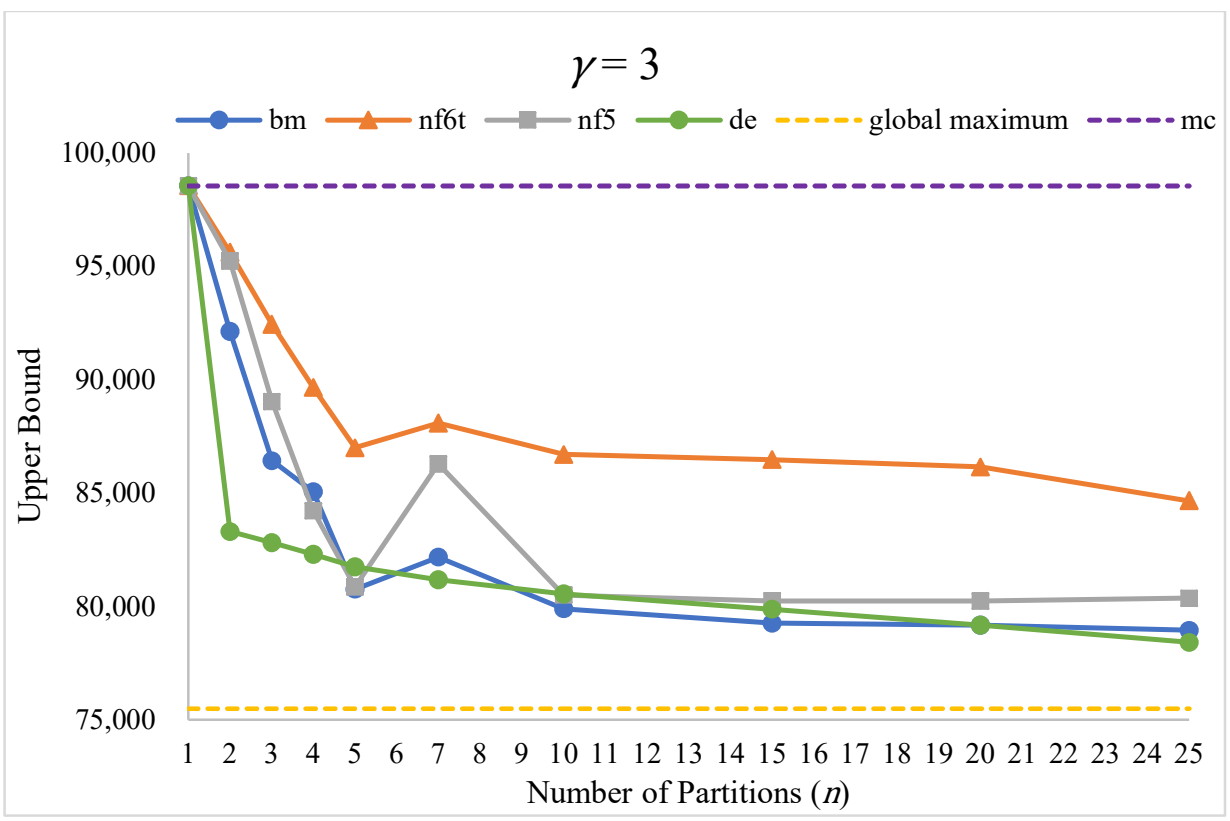

Figure 9. Convergence-indicating curves of bm, de, $\mathbf{n f 5}$, and $\mathbf{n f 6 t}$ relaxation schemes for $\gamma=$ 3. 


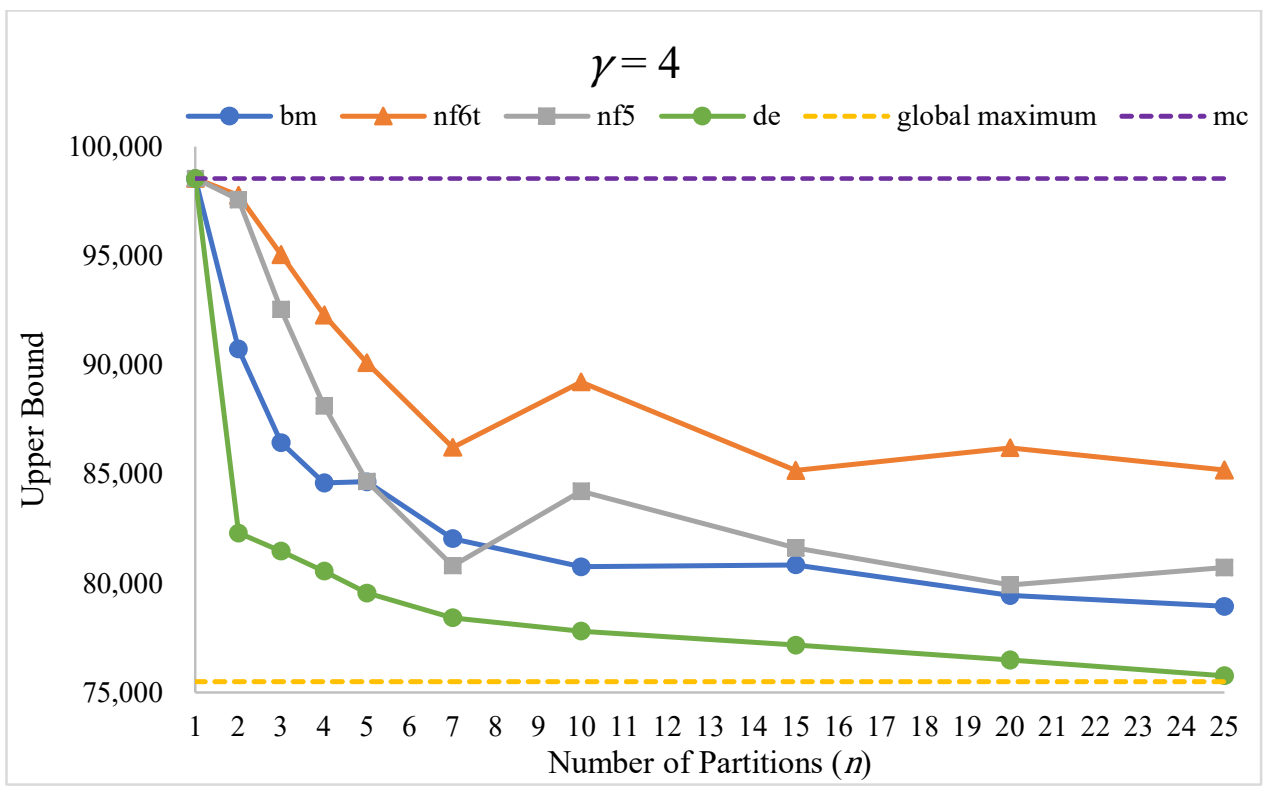

Figure 10. Convergence-indicating curves of $\mathbf{b m}, \mathbf{d e}, \mathbf{n f 5}$, and $\mathbf{n f 6 t}$ relaxation schemes for $\gamma=$ 4.

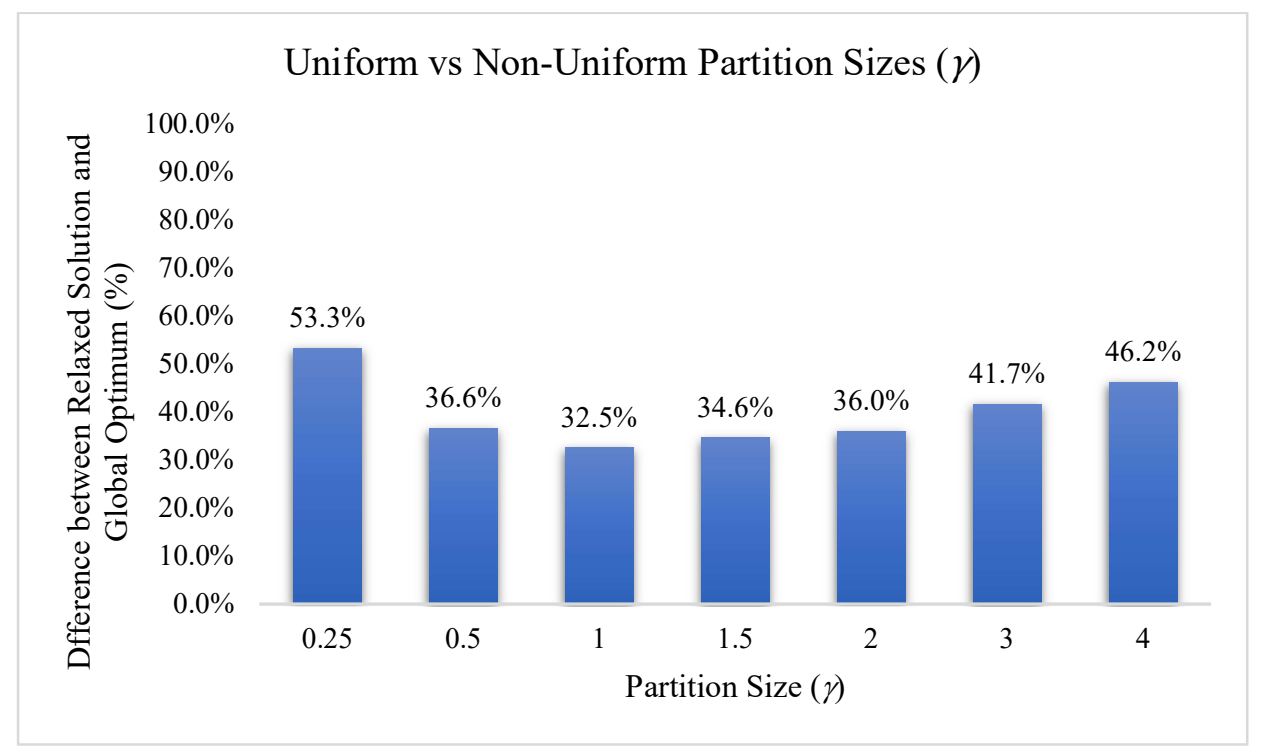

Figure 11. Performance of uniform $(\gamma=1)$ and non-uniform partition sizes $(\gamma \neq 1)$.

\section{CONCLUDING REMARKS}

In this work, we apply piecewise-linear relaxation schemes to convexify bilinear terms in a refinery planning NLP model and study the resulting performance by varying the number and 
size of partitions. As alternatives to conventional measures (e.g., computational time or optimality gap, which are more applicable to large scale industrial-size problems), we consider certain indicators to compare the schemes in terms of convergence and partitioning behavior. Our study encounters a limit on the number of partitions that contributes to relaxation tightness which does not necessarily correspond to a larges number of partitions. Further, the computational results show better performance in using uniform partition sizes. These results are largely consistent with the literature.

\section{ASSOCIATED CONTENT}

Mathematical formulation of the refinery planning NLP model is available as Supplementary Material.

\section{ACKNOWLEDGEMENT}

This work is partly supported by funding from Universiti Teknologi PETRONAS Graduate Assistantship Scheme through grant under identification (ID) 20000015 for Zaid Ashraf Rana.

\section{NOMENCLATURE}

Sets and indices

$\begin{array}{ll}U & \text { set of process unit } u \\ P & \text { set of CDU fraction } p \\ N & \text { set of gridpoints for segment } n\end{array}$

Parameters

$\begin{array}{ll}\gamma & \text { segment size (i.e., length) } \\ M & \text { big- } M \text { parameter } \\ k_{n, p} & \text { gridpoint for segment } n\end{array}$


$q_{n} \quad$ length of segment $n$

Continuous variables

$\begin{array}{ll}L_{u} & \text { load of process unit } u \\ W_{p} & \text { weight transfer ratio of fraction } p \text { (for CDU) } \\ d W & \text { deviation of partitioned variable in bilinear term from lower bound } \\ d U, d V & \text { auxiliary variable for relaxation } \\ \omega_{u, p} & \text { variable for reformulation to replace bilinear term }\end{array}$

Binary variables

$\lambda \quad$ disjunction in $\mathbf{b m}$ scheme

$\theta \quad$ disjunction in $\mathbf{n f 5}$ and $\mathbf{n f 6 t}$ formulations

\section{REFERENCES}

(1) Aspen Technology Aspen Pims and Aspen Pims-Ao. http://www.aspentech.com/brochures/aspen_pims_ao.pdf (accessed August 15).

(2) Honeywell Symphonite Rpms (Refining and Petrochemical Modeling System).

(3) Haverly Systems Grtmps. http://www.haverly.com/main-products/13-products/9-grtmps (accessed May 9).

(4) Barsamian, A. In Fundamentals of Supply Chain Management for Refining, IBC Asia Oil \& Gas SCM Conference Proceedings, 2001.

(5) Andrade, T.; Ribas, G.; Oliveira, F., A Strategy Based on Convex Relaxation for Solving the Oil Refinery Operations Planning Problem. Industrial \& Engineering Chemistry Research 2016, 55 (1), 144-155.

(6) Rigby, B.; Lasdon, L. S.; Waren, A. D., The Evolution of Texaco's Blending Systems: From Omega to Starblend. Interfaces 1995, 25 (5), 64-83.

(7) Moro, L. F. L.; Zanin, A. C.; Pinto, J. M., A Planning Model for Refinery Diesel Production. Computers \& Chemical Engineering 1998, 22, S1039-S1042.

(8) Steinschorn, D.; Hofferl, F. In Refinery Scheduling Using Mixed Integer Lp and Dynamic Recursion, National Petroleum Refiners Association-Publications-All Series, 1997.

(9) Khor, C. S.; Varvarezos, D., Petroleum Refinery Optimization. Optimization and Engineering 2016, 18 (4), 943-989.

(10) Khor, C. S.; Elkamel, A., Roles of Computers in Petroleum Refineries. In Handbook of Petroleum Refining and Natural Gas Processing, Riazi, M. R.; Eser, S.; Diez, J. L. P.; Agrawal, S. S., Eds. ASTM International: Conshohocken, PA, USA, 2013; Vol. Manual 58, pp 685 700 .

(11) Watkins, R. N., Petroleum Refinery Distillation. Gulf Publishing Company, Book division: Houston (Tex.); London; Paris, 1979. 
(12) Li, X.; Lin, C.; Wang, L.; Li, H., Exergy Analysis of Multi-Stage Crude Distillation Units. Frontiers of Chemical Science and Engineering 2013, 7 (4), 437-446.

(13) Geddes, R. L., A General Index of Fractional Distillation Power for Hydrocarbon Mixtures. AIChE Journal 1958, 4 (4), 389-392.

(14) Menezes, B. C.; Kelly, J. D.; Grossmann, I. E., Improved Swing-Cut Modeling for Planning and Scheduling of Oil-Refinery Distillation Units. Industrial \& Engineering Chemistry Research 2013, 52 (51), 18324-18333.

(15) Brooks, R.; Walsem, F. D.; Drury, J., Choosing Cutpoints to Optimize Product Yields. Hydrocarbon Processing 1999, 78, 53-60.

(16) Khor, C. S.; Elkamel, A.; Ponnambalam, K.; Douglas, P. L., Two-Stage Stochastic Programming with Fixed Recourse Via Scenario Planning with Economic and Operational Risk Management for Petroleum Refinery Planning under Uncertainty. Chemical Engineering and Processing 2008, 47 (9-10), 1744-1764.

(17) Pinto, J. M.; Joly, M.; Moro, L. F. L., Planning and Scheduling Models for Refinery Operations. Computers \& Chemical Engineering 2000, 24 (9), 2259-2276.

(18) Alattas, A. M.; Grossmann, I. E.; Palou-Rivera, I., Integration of Nonlinear Crude Distillation Unit Models in Refinery Planning Optimization. Industrial \& Engineering Chemistry Research 2011, 50 (11), 6860-6870.

(19) Trierwiler, Advances in Crude Oil Lp Modeling. National Petrochemical \& Refiners Association: 2001.

(20) Alhajri, I.; Elkamel, A.; Albahri, T.; Douglas, P. L., A Nonlinear Programming Model for Refinery Planning and Optimisation with Rigorous Process Models and Product Quality Specifications. International Journal of Oil, Gas and Coal Technology 2008, 1 (3), 283-307.

(21) Alhajri, I.; Saif, Y.; Elkamel, A.; Almansoori, A., Overall Integration of the Management of $\mathrm{H} 2$ and $\mathrm{Co} 2$ within Refinery Planning Using Rigorous Process Models. Chemical Engineering Communications 2013, 200 (1), 139-161.

(22) Zhang, J.; Zhu, X.; Towler, G., A Level-by-Level Debottlenecking Approach in Refinery Operation. Industrial \& Engineering Chemistry Research - IND ENG CHEM RES 2001, 40.

(23) Li, W.; Hui, C.-W.; Li, A., Integrating Cdu, Fcc and Product Blending Models into Refinery Planning. Computers \& Chemical Engineering 2005, 29 (9), 2010-2028.

(24) Menezes, B. C.; Moro, L. F. L.; Lin, W. O.; Medronho, R. A.; Pessoa, F. L. P., Nonlinear Production Planning of Oil-Refinery Units for the Future Fuel Market in Brazil: Process Design Scenario-Based Model. Industrial \& Engineering Chemistry Research 2014, 53 (11), 43524365.

(25) Guerra, O. J.; Le Roux, G. A. C., Improvements in Petroleum Refinery Planning: 1. Formulation of Process Models. Industrial \& Engineering Chemistry Research 2011, 50 (23), 13403-13418.

(26) Guerra, O. J.; Le Roux, G. A. C., Improvements in Petroleum Refinery Planning: 2. Case Studies. Industrial \& Engineering Chemistry Research 2011, 50 (23), 13419-13426.

(27) Li, J.; Xiao, X.; Boukouvala, F.; Floudas, C. A.; Zhao, B. G.; Du, G. M.; Su, X.; Liu, H. W., Data-Driven Mathematical Modeling and Global Optimization Framework for Entire Petrochemical Planning Operations. AIChE Journal 2016, 62 (9), 3020-3040.

(28) Alattas, A. M.; Grossmann, I. E.; Palou-Rivera, I., Refinery Production Planning: Multiperiod Minlp with Nonlinear Cdu Model. Industrial \& Engineering Chemistry Research 2012, 51 (39), 12852-12861.

(29) Castillo Castillo, P.; Castro, P. M.; Mahalec, V., Global Optimization Algorithm for Large-Scale Refinery Planning Models with Bilinear Terms. Industrial \& Engineering Chemistry Research 2017, 56 (2), 530-548. 
(30) McCormick, G. P., Computability of Global Solutions to Factorable Nonconvex Programs: Part I-Convex Underestimating Problems. Mathematical programming 1976, 10 (1), 147-175.

(31) Al-Khayyal, F. A.; Falk, J. E., Jointly Constrained Biconvex Programming. Mathematics of Operations Research 1983, 8 (2), 273-286.

(32) Misener, R.; Floudas, C. A., Antigone: Algorithms for Continuous/Integer Global Optimization of Nonlinear Equations. Journal of Global Optimization 2014, 59 (2), 503-526.

(33) Li, J.; Xiao, X.; Boukouvala, F.; Floudas, C. A.; Zhao, B.; Du, G.; Su, X.; Liu, H., DataDriven Mathematical Modeling and Global Optimization Framework for Entire Petrochemical Planning Operations. AIChE Journal 2016, 62 (9), 3020-3040.

(34) Meyer, C. A.; Floudas, C. A., Global Optimization of a Combinatorially Complex Generalized Pooling Problem. AIChE Journal 2006, 52 (3), 1027-1037.

(35) Karuppiah, R.; Grossmann, I. E., Global Optimization for the Synthesis of Integrated Water Systems in Chemical Processes. Computers \& Chemical Engineering 2006, 30 (4), 650673.

(36) Wicaksono, D. S.; Karimi, I. A., Piecewise Milp under- and Overestimators for Global Optimization of Bilinear Programs. AIChE Journal 2008, 54 (4), 991-1008.

(37) Gounaris, C. E.; Misener, R.; Floudas, C. A., Computational Comparison of Piecewise-Linear Relaxations for Pooling Problems. Industrial \& Engineering Chemistry Research 2009, 48 (12), 5742-5766.

(38) Hasan, M. M. F.; Karimi, I. A., Piecewise Linear Relaxation of Bilinear Programs Using Bivariate Partitioning. AIChE Journal 2009, 56 (7), 1880-1893.

(39) Misener, R.; Floudas, C. A., Advances for the Pooling Problem: Modeling, Global Optimization, and Computational Studies. Appl. Comput. Math 2009, 8 (1), 3-22.

(40) Nagarajan, H.; Lu, M.; Wang, S.; Bent, R.; Sundar, K., An Adaptive, Multivariate Partitioning Algorithm for Global Optimization of Nonconvex Programs. Journal of Global Optimization 2019, 74 (4), 639-675.

(41) Tawarmalani, M.; Ahmed, S.; Sahinidis, N. V., Global Optimization of 0-1 Hyperbolic Programs. Journal of Global Optimization 2002, 24 (4), 385-416.

(42) Tawarmalani, M.; Sahinidis, N. V., Convexification and Global Optimization in Continuous and Mixed-Integer Nonlinear Programming: Theory, Algorithms, Software, and Applications. Kluwer Academic Publishers: Dordrecht, 2002; Vol. 65, p 504.

(43) Grossmann, I. E.; Lee, S., Global Optimization of Nonlinear Generalized Disjunctive Programming with Bilinear Equality Constraints: Applications to Process Networks. Computers \& Chemical Engineering 2003, 27 (11), 1557-1575.

(44) Hasan, M. M. F.; Karimi, I. A., Piecewise Linear Relaxation of Bilinear Programs Using Bivariate Partitioning. AIChE Journal 2010, 56 (7), 1880-1893.

(45) Uribe-Rodriguez, A.; Castro, P. M.; Gonzalo, G.-G.; Chachuat, B., Global Optimization of Large-Scale Miqcqps Via Cluster Decomposition: Application to Short-Term Planning of an Integrated Refinery-Petrochemical Complex. Computers \& Chemical Engineering 2020, 140, 106883.

(46) Yang, H.; Bernal, D. E.; Franzoi, R. E.; Engineer, F. G.; Kwon, K.; Lee, S.; Grossmann, I. E., Integration of Crude-Oil Scheduling and Refinery Planning by Lagrangean Decomposition. Computers \& Chemical Engineering 2020, 138, 106812.

(47) Horst, R.; Tuy, H., Global Optimization: Deterministic Approaches. 3rd ed.; SpringerVerlag: Berlin, 1996.

(48) Nemhauser, G.; Wolsey, L., Integer and Combinatorial Optimization. Wiley: New York, 1988. 
(49) Tawarmalani, M.; Sahinidis, N. V., Global Optimization of Mixed-Integer Nonlinear Programs: A Theoretical and Computational Study. Mathematical Programming 2004, 99 (3), 563-591.

(50) Tawarmalani, M.; Sahinidis, N. V., A Polyhedral Branch-and-Cut Approach to Global Optimization. Mathematical Programming 2005, 103 (2), 225-249.

(51) Sahinidis, N. V.; Tawarmalani, M., Baron 7.2.5: Global Optimization of Mixed-Integer Nonlinear Programs, User's 2005. http://www.andrew.cmu.edu/user/ns1b/baron/bibliography.html (accessed May 29, 2011).

(52) Zamora, J. M.; Grossmann, I. E., Continuous Global Optimization of Structured Process Systems Models. Computers \& Chemical Engineering 1998, 22 (12), 1749-1770.

(53) Misener, R.; Floudas, C., Glomiqo: Global Mixed-Integer Quadratic Optimizer. Journal of Global Optimization 2013, 57 (1), 3-50.

(54) Neumaier, A., Complete Search in Continuous Global Optimization and Constraint Satisfaction. Acta Numerica 2004, 13, 271-369.

(55) McCormick, G. P., Computability of Global Solutions to Factorable Nonconvex Programs .1. Convex Underestimating Problems. Mathematical Programming 1976, 10 (2), 147-175.

(56) Smith, E. M. B.; Pantelides, C. C., A Symbolic Reformulation/Spatial Branch-and-Bound Algorithm for the Global Optimisation of Nonconvex Minlps. Computers \& Chemical Engineering 1999, 23 (4), 457-478.

(57) Faria, D. C.; Bagajewicz, M. J., Global Optimization of Water Management Problems Using Linear Relaxation and Bound Contraction Methods. Industrial \& Engineering Chemistry Research 2011, 50 (7), 3738-3753.

(58) Faria, D. C.; Bagajewicz, M. J., Novel Bound Contraction Procedure for Global Optimization of Bilinear Minlp Problems with Applications to Water Management Problems. Computers \& Chemical Engineering 2011, 35 (3), 446-455.

(59) Faria, D. C.; Bagajewicz, M. J., A New Approach for Global Optimization of a Class of Minlp Problems with Applications to Water Management and Pooling Problems. AIChE Journal 2012, 58 (8), 2320-2335.

(60) Faria, D. C.; Bagajewicz, M. J., Global Optimization Based on Subspaces Elimination: Applications to Generalized Pooling and Water Management Problems. AIChE Journal 2012, $58(8), 2336-2345$. 